N. E. Piskunov, W. W. Weiss, D. F. Gray, eds.

\title{
Round Table Summary: \\ Stellar Interferometry as a Tool to Investigate Atmospheres and to Compare Observations with Models
}

\author{
Markus Wittkowski \\ European Southern Observatory, Casilla 19001, Santiago 19, Chile, \\ mwittkow@eso.org
}

\begin{abstract}
Long-baseline interferometry at optical and near-infrared wavelengths is an emerging technology which is quickly becoming a useful tool to investigate stellar atmospheres and to compare observations with models. Stellar atmosphere models have so far mainly been constrained by comparisons with stellar spectra which are integrated over the stellar disks. Interferometric observations provide spatially and spectrally resolved information and can thus provide important complementary observational information which can be compared to model predictions. Here, I summarize the different aspects on this topic which were discussed at a round table on Thursday, June 20, 2002, during IAU Symposium 210. This summary gives an overview on discussed interferometric facilities and techniques, concepts to study atmospheres by optical interferometry, and particular classes of objects. We conclude that more frequent interactions between the efforts of atmosphere modelling and interferometric observations promise to lead to increased confidence in stellar model atmospheres and to further advancement of the field in the next years.
\end{abstract}

\section{Introduction}

Long-baseline interferometry at optical and near-infrared wavelengths is an emerging technology which is quickly becoming a useful tool to investigate stellar atmospheres and to compare observations with models. A round table on this topic was held on June 20, 2002, during the IAU Symposium 210 "Modelling of stellar atmospheres". The goal of this round table was to discuss and stimulate interactions between observations obtained by optical interferometers and theoretical modelling in the field of stellar atmospheres. Here, I summarize aspects that were discussed at the round table, or during the course of the symposium. This summary is based on the individual contributions which can be found in this volume, supplemented by some further literature that was cited at the symposium, without any claim of completeness. This round table summary is, hence, not intended to be a complete review of the field. Optical interferometry, including interferometric techniques as well as scientific studies, have recently been reviewed by Shao \& Colavita (1992), Quirrenbach (2001), and Baldwin \& Haniff (2002). OLBIN (optical long baseline interferometry news, edited by P. Lawson, and available at olbin.jpl.nasa.gov) is an excellent source of up-to- 
date information in the field of optical interferometry. Circumstellar envelopes are also studied by maser observations using radio interferometry (Humphreys, this volume). However, radio interferometry was not part of this round table discussion.

In general, information on stellar atmospheres using optical long-baseline interferometry has so far been derived by comparing model predictions to observations and measurements of

- angular sizes of stars and derived absolute radii and effective temperatures

- center-to-limb intensity variations

- asymmetric shapes of stellar surfaces

- horizontal surface inhomogeneities

- circumstellar matter.

These measurements have often been temporally or spectrally resolved, which leads to additional insights into the atmospheres' structures and dynamics.

In the following, topics of discussion are summarized in the contexts of (1) interferometric facilities and techniques, (2) concepts to study stellar atmospheres, which are applicable to different object classes, or (3) certain classes of objects. There is, however, overlap between these categories.

\section{Interferometric techniques and facilities}

Interferometric facilities Results, modelling of results, or predictions using the following interferometric facilities were presented at the symposium.

\begin{tabular}{|c|c|c|}
\hline Name & Location & References in this volume \\
\hline COAST & Cambridge, UK & $\begin{array}{l}\text { Freytag \& Mizuno-Wiedner, Freytag \& } \\
\text { Finnson, van Belle et al. (b). }\end{array}$ \\
\hline GI2T & Calern, France & $\begin{array}{l}\text { Dominiciano et al., Jankov et al., Stee \& } \\
\text { Bitter. }\end{array}$ \\
\hline IOTA & Mt. Hopkins, USA & van Belle et al. (b). \\
\hline MARK III & Mt. Wilson, USA & Quirrenbach \& Aufdenberg. \\
\hline NPOI & Flagstaff, USA & Wittkowski. \\
\hline PTI & Mt. Palomar, USA & van Belle et al. $(a, b)$. \\
\hline KECK & M. Kea, USA & van Belle et al. $(a, b)$ \\
\hline VLTI & Paranal, Chile & $\begin{array}{l}\text { Dominiciano et al., Jankov et al., Stee \& } \\
\text { Bitter, Wittkowski, Wittkowski et al. }\end{array}$ \\
\hline
\end{tabular}

Further operating interferometric facilities include

CHARA Mt. Wilson, USA

ISI Mt. Wilson, USA

SUSI Narrabri, Australia

For further information on these facilities, see the references mentioned above, 
Baldwin \& Haniff (2002), Quirrenbach (2001), or OLBIN. The above mentioned interferometric facilities are generally available to the stellar atmosphere community through acceptance of observing proposals and/or collaborations, with the exception of the MARK III, which is not operational anymore. Detailed procedures and priorities for scientific topics differ. For instance, ESO currently accepts scientific proposals on all fields from the international community to be carried out on a shared-risk basis with the VLTI and the near-infrared K-band commissioning instrument VINCI. A first call for proposals was issued for Period 70 (October 2002 to March 2003) and a second call for Period 71 (April 2003 to October 2003). Calls for proposals for the scientific instruments MIDI (mid-infrared) and AMBER (near-infrared) can be expected for 2003/2004. In general, due to the limiting magnitudes, interferometers are mostly used for stellar astrophysics, and hence stellar atmospheres. Additional priorities of large facilities using 8-10m class telescopes (Keck, VLTI, LBT) include planet searches and active galactic nuclei.

Interferometric observables The primary observables of an interferometer are the amplitude and phase of the complex visibility function, which is the Fourier transform of the object intensity distribution (van Cittert-Zernike Theorem). In presence of atmospheric turbulence, usually only the squared visibility amplitudes and the triple products are accessible by optical and near-infrared interferometers. A triple product is the product of three complex visibilities corresponding to baselines that form a triangle. The phase of the triple product, the closure phase, is free of atmospheric phase noise (Jennison 1958). Thus, the measurement of triple products is a method to obtain phase information of the object intensity distribution, i.e. allows us to detect deviations from point symmetry. Observational results that were discussed at the symposium make use of squared visibility amplitudes (Quirrenbach \& Aufdenberg, van Belle et al. (a/b), Wittkowski, Wittkowski et al.). The COAST images of Betelgeuse (Buscher et al. 1990), modelled by Freytag \& Finnson and Freytag \& Mizuno-Wiedner, as well as the NPOI limb-darkening measurements presented by Wittkowski make in addition use of triple products. The vast majority of interferometric studies use measurements of relatively large (squared) visibility amplitudes before the first minimum of the visibility function. Since this means that less than one resolution element across the stellar disk is obtained, one parameter, i.e. the diameter of the disk, is derived. These diameter measurements can however be done at different position angles in order to detect asymmetric shapes of the stellar disks (van Belle et al. (a,b)), at different wavelengths (Quirrenbach \& Aufdenberg, Wittkowski), or at different time periods to detect for instance stellar pulsations. Interferometric observations of more than one resolution element across the stellar disks are difficult to perform, and hence rare. The long baselines needed to obtain this resolution, i.e. to resolve the stellar disk, also produce very low visibility amplitudes. These low visibility amplitudes correspond to vanishing fringe contrasts. Hence, the corresponding fringes are difficult to be detected, tracked, and recorded. In addition, the data reduction of low visibility values is often a special challenge as well, since the contributions of photon noise and detection bias terms might become dominant. Such 
observations and the successful compensation of bias terms are discussed in this volume by Wittkowski.

In addition to visibility amplitudes and triple products, the phase of the complex visibility is an interferometric observable as well, as mentioned above. If atmospheric and instrumental noise can be controlled or compensated, for example by phase referencing using a reference star within the isoplanatic patch or by using the same source at another wavelength, the relative position of the object on the sky can be derived with an accuracy which is much higher than the diffraction limit. If the position of the photocenter of the object depends on the wavelength, such differential phase measurements can be used to derive information on the spatial object brightness distribution. Information on stellar diameters, stellar rotation, and starspots can be derived. This technique was described by Beckers (1982), Chelli \& Petrov (1995), Jankov et al. (2001), and is discussed in this volume by Dominiciano de Souza et al. and Jankov et al. Jankov et al. discuss in particular the imaging potential of a tomographic technique which combines time-resolved spectroscopy (Doppler Imaging) and differential interferometry.

Simulations of complete atmospheres of stars, such as the radiation hydrodynamics simulations of Betelgeuse presented by Freytag \& Finnson and Freytag \& Mizuno Wiedner would ideally be compared with high-resolution images of stellar surfaces derived by interferometric observations. If modulus and phase of the complex visibility values are derived, the object intensity distribution can in principle be directly reconstructed from interferometric data using imaging techniques which effectively interpolate the limited coverage of the Fourier-plane. A recent review describing the image fidelity using optical interferometers can be found in Baldwin \& Haniff (2002). In order to reconstruct an image of reasonable fidelity, it is intuitively understandable that the number of visibility data points has to be at least as large as the number of unknowns, i.e. the pixel intensities of the image. Furthermore, despite the ability of imaging algorithms to effectively interpolate the sparse aperture data, the aperture has to be filled as uniformly as possible in order to avoid strong artifacts of the PSF such as sidelobes. This means that many very low visibility values at several lobes of the visibility function and at different azimuth angles have to be measured. As a result, images will probably be limited to a few pixels (about $3 \times 3-5 \times 5 \mathrm{px}$.) in the next years. It was discussed that model fitting, for instance fitting of parameters that describe asymmetries as a function of time, might be a better choice for first attempts.

Calibration Interferometric observations are generally limited by the calibration of the atmospheric and instrumental transfer function, i.e. the instrumental visibility response to a point source. This quantity is, dependent on instrument and site, observed to be dependent on different parameters as for instance seeing, atmospheric coherence time, or zenith angle of the source. The transfer function is usually calibrated by observations of an unresolved star or a star with well-known diameter. Since, especially if long baselines are used, almost all calibrator stars are at least slightly resolved, the uncertainty of the knowledge of the diameter of the calibrator star becomes often a major limitation (e.g. Percheron et al. 2002). In addition, the diameter of the calibrator star is often 
obtained at a wavelength different from that of the new experiment. In this case, model atmospheres need to be used to predict the interferometric transfer function. This can best be done by using the Rosseland mean angular diameter. Then, the model atmosphere is needed to derive the transfer function based on the Rosseland mean angular diameter, or to transform the Rosseland mean angular diameter into a more practical uniform disk diameter at the wavelength of the experiment. As a result, calibration is a topic for which optical interferometry relies on stellar atmosphere modelling, rather than providing observational constraints for models.

\section{Concepts to study stellar atmospheres}

The following concepts to study stellar atmospheres were discussed. These concepts are usually applicable to different classes of objects.

Diameters, radii, effective temperatures The vast majority of interferometric measurements have so far directly derived only the first stellar surface structure parameter, which is the angular size of the star. The combination of the angular diameter with the bolometric flux is the most direct method to obtain the effective temperature of the star. This is an important constraint for comparisons of model atmospheres with stellar spectra, since it fixes one free parameter. Another diameter that can be derived from the angular diameter is the absolute radius of the star if its distance is known, e.g. by using the Hipparcos parallax. Further fundamental stellar parameters have been derived from observations of binary systems (e.g. Hummel et al. 1995). The stellar diameter is usually obtained using a uniform disk model. The uniform disk diameter is wavelength-dependent due to the limb-darkening effect (see, e.g. Quirrenbach et al. 1996, Wittkowski et al. 2001). Stellar model atmospheres are used to transform wavelength-dependent uniform disk diameters at the observational wavelength into wavelength-independent limb-darkened diameters. The observed relative variation of uniform disk diameters as a function of wavelength can also be compared to model predictions of this wavelength dependence, and hence be used to test model atmospheres. Quirrenbach \& Aufdenberg present in this volume uniform disk diameter measurements of 47 cool giants with the Mark III Interferometer on Mt. Wilson, California in a strong TiO band and in the continuum. They reproduce the diameter ratios by PHOENIX state of the art model atmospheres (Hauschildt et al. 1997; Baron et al., this volume) and derive important constraints for these models. This concept can be used for many different stars using modern interferometers equipped with instruments that allow spectral resolution, as for instance the VLTI with the AMBER instrument at near-infrared wavelengths. Van Belle et al. (b) discuss in this volume wavelength-dependent diameter measurements of Mira stars. Narrowband diameters of oxygen-rich and carbon-rich Miras and non-Mira stars obtained with the PTI are presented; Mira diameter measurements obtained by different interferometers are reviewed.

Asymmetric shapes of stellar surfaces Interferometric measurements can also derive asymmetric shapes of stellar surfaces if baselines of different orien- 
tation are used. Van Belle et al. (a) present in this volume the recent detection of an oblate photosphere of a fast rotator, the main sequence star Altair (see also van Belle et al. 2001). The theoretical description and simulations of observations of stellar rotation are also discussed in this volume by Dominiciano de Souza et al. and Jankov et al. (see also Domiciano de Souza et al. 2002, Jankov et al. 2001). Van Belle et al. (b) discuss in this volume that also for Mira stars indications of departures from spherical symmetry are beginning to be observed as increasingly rich image information is obtained by a new generation of interferometers. Mira stars rotate slowly, and hence, stellar rotation is an unlikely cause for these asymmetries. The nature of the asymmetry of surfaces of AGB stars is not well understood.

Intensity profiles and limb-darkening Optical interferometry has proven its capability to go beyond the measurement of diameters, and to measure additional surface structure parameters. Through the direct measurement of stellar limb-darkening, interferometry tests the wavelength-dependent intensity profile across the stellar disk. Unfortunately, direct measurements of the limb-darkening effect are rare because of the observational difficulties mentioned above. While diameters have so far been obtained for several hundred stars with interferometric and lunar occultation techniques, limb-darkening has been directly observed for only a very limited number of stars (Hanbury Brown et al. 1974, Haniff et al. 1995, Quirrenbach et al. 1996, Burns et al. 1997, Hajian et al. 1998, Wittkowski et al. 2001). Wittkowski (this volume) present multi-wavelength limb-darkening measurements on the giant star $\gamma$ Sge, obtained with the NPOI, which succeeded not only in discriminating between uniform disks and limbdarkened disks, but also in constraining Kurucz model atmosphere parameters (Wittkowski et al. 2001). First VLTI measurements of visibility values beyond the first minimum were shown as well. Confronting models with both measured stellar spectra and measured intensity profiles at the same time is a strong test for the radiation fields predicted by atmosphere models (see, e.g. Aufdenberg \& Hauschildt 2003). Considering the rapid evolution of interferometry, it can be expected that more limb-darkening parameters of a wider variety of stars can be measured soon with high spectral resolution.

Surface features The final goal is the reconstruction of images of stellar surfaces with several resolution elements across the stellar disk, revealing both the overall center-to-limb intensity variation and additional horizontal inhomogeneities as warm or cool starspots. These inhomogeneities may be caused by photospheric convection, magnetic fields, or abundance inhomogneities. It is expected that starspots on relatively large stars can directly be detected and its parameters be constrained using squared visibility amplitudes and triple products obtained with the VLTI with the AMBER instrument (see Wittkowski, this volume; Wittkowski et al. 2002). Jankov et al. discuss in this volume the potential to combine the techniques of stellar differential (phase) interferometry and Doppler imaging in order to constrain surface features on smaller rotating stars. Parameters describing horizontal surface inhomogeneities can then be compared to simulations of entire stellar atmospheres. Freytag \& Mizuno-Wiedner and Freytag \& Finnson (this volume) present $3 \mathrm{D}$ radiation hydrodynamic simula- 
tions of the envelope and atmosphere of a red supergiant, in order to model observational data of Betelgeuse.

\section{Classes of objects}

A few particular classes of objects were discussed in the course of the symposium, for which comparisons of models with interferometric observational data seem to be promising.

Red giants and supergiants Red giant and supergiant stars have so far been the prime target for interferometric observations. This is caused by their large apparent sizes, and their brightness, especially at near-infrared wavelengths. Surface features have been detected on the surfaces of the apparently largest supergiants $\alpha$ Orionis, $\alpha$ Scorpii and $\alpha$ Herculi (e.g. Buscher et al. 1990, Burns et al. 1997, Young et al. 2000). Models to describe such surface features are presented by Freytag \& Mizuno-Wiedner and Freytag \& Finnson (this volume).

AGB stars and Mira stars The evolution of late-type stars along the asymptotic giant branch (AGB) is characterized by high luminosities and low effective temperatures, and accompanied by significant mass loss to the circumstellar environment with mass-loss rates of up to $10^{-7}-10^{-4} \mathrm{M}_{\odot} /$ year. AGB stars with masses of the order of $1 \mathrm{M}_{\odot}$ become unstable to large amplitude radial pulsations and become Mira variable stars. The processes of mass-loss and pulsation are essential for our understanding of late stages of stellar evolution, but are still a matter of debate (see the excellent keynote presentation by Woitke in this volume). Atmosphere models need to couple time-dependent dynamics, radiative transfer, chemistry, and dust formation (Woitke, Höfner et al., both in this volume). Optical interferometry can provide important observational constraints by measuring the intensity profile across the stellar disks, which are often observed to be very different from uniform disks (Wittkowski et al., this volume), the time/stellar phase dependent diameter variations, and absolute radii indicating the mode of pulsation (see, e.g. Hofmann et al. 2002). Interferometric observations of Mira stars have recently been performed by COAST (Young et al. 2000), IOTA (van Belle et al. 2002, Hofmann et al. 2002), and PTI (Thompson et al. 2002). These observations are reviewed by van Belle et al. in this volume. Wittkowski et al. present first preliminary results of Mira observations with the VLTI and the VINCI commissioning instrument.

Small cool stars Ludwig (this volume) present model atmospheres for mid Mtype main-, as well as pre-main-sequence objects. These model atmospheres are characterized by low effective temperatures and high surface gravities. Because of the rather small angular sizes, these objects are difficult for interferometric observations. However, first measurements of diameters of $\mathrm{M}$ dwarfs succeeded already (Lane et al. 2001, Segransan et al. 2003). These observations provide an empirical mass-radius relation, and can thus provide observational constraints for atmosphere models of this parameter space (low masses, low effective temperatures, high surface gravities). 
Winds from Be stars Classical Be stars are rapidly rotating hot giant or main-sequence stars. They show asymmetric circumstellar winds in the light of emission lines. Stee \& Bitter (2001, and this volume) present theoretical models of Be star winds, and predict constraints by near-infrared and visible interferometric observations. Optical interferometry has already been successfully used to study Be star winds (Thom et al. 1986, Quirrenbach et al. 1993, Stee et al. 1995). Upcoming facilities with high spatial and high spectral resolution as for instance VLTI with the AMBER instrument are expected to provide stronger constraints on Be star winds.

\section{Concluding remarks}

Stellar atmosphere models are still mostly constrained by observations of stellar spectra only, which are integrated over the stellar disks. This round table discussion has shown that optical interferometry has already proven its ability to provide important additional observational constraints by spatially resolved observations of stellar disks and circumstellar matter. The comparison of model atmospheres with both stellar spectra and interferometric observations is a strong test and leads as well to increased confidence in these models. Observational results for different object classes and for different parameter spaces of stellar model atmospheres have been presented in the course of this symposium. Optical interferometry is currently rapidly evolving, and simulations show that stronger constraints for more stars can be expected soon. Most progress in this field can be made by increasing interactions between the efforts of theoretical atmosphere modelling and interferometric observations.

Acknowledgments. I am grateful to the speakers of this round table discussion, Bernd Freytag, Robert Kurucz, Andreas Quirrenbach, Slobodan Jankov, Philippe Stee, Gerard van Belle, for their contributions. I thank the SOC for allocating time for a round table on stellar interferometry, and for the invitation as a round table organizer.

\section{References}

Aufdenberg J., Hauschildt P.H., 2003, Proc. SPIE 4838, in press

Baldwin J.E., Haniff C.A., 2002, Phil. Trans. A, 360, 969

Baron E., Hauschildt P.H., Allard F., et al., this volume

Beckers J.M., 1982, Optica Acta 29, 361

Burns D., Baldwin J.E., Boysen R.C., et al., 1997, MNRAS 245, 7

Buscher D.F. Haniff C.A., Baldwin J.E., Warner P.J., 1990, MNRAS 245, 7p

Dominiciano de Souza A., Vakili F., Jankov S., et al., 2002, A\&A 393, 345

Dominiciano de Souza A., Zorec J., Jankov S., Vakili F., Petrov R.G., Abe L., this volume

Freytag B., Finnson S., this volume

Freytag B., Mizuno-Wiedner M., this volume

Hajian A.R., Armstrong J.T., Hummel C.A., et al., 1998, ApJ 496, 484 
Hanbury Brown R., Davis J., Lake R.J.W., et al. 1974, MNRAS 167, 475

Haniff C.A., Scholz M., Tuthill P.G., 1995, MNRAS 276, 640

Hauschildt P.H., Baron E., Allard F., 1997, ApJ 483, 390

Hofmann K.-H., et al., 2002, New Astronomy 7, 9

Höfner S., Sandin C., Aringer B., et al., this volume

Hummel C.A., Armstrong J.T., Buscher D.F., et al., 1995, AJ 110, 376

Humphreys E.M.L., 2001, this volume

Jankov S., Vakili F., Domiciano de Souza A., this volume

Jankov S., Vakili F. Dominiciano de Souza Jr., Janot-Pacheco E., 2001, A\&A 377,721

Jennison R.C., 1958, MNRAS 118, 276

Kurucz R., 1993, Kurucz CD ROMs, Cambridge, Mass.: Smithsonian Astrophysical Observatory

Kurucz R.L., this volume

Lane B.F., Boden A.F., Kulkarni S.R., 2001, ApJ 551, L81

Ludwig H.-G., this volume

Percheron I., Richichi A., Wittkowski M., 2003, Proc. SPIE 4838, in press

Quirrenbach A., Mozurkewich D., Armstrong T., et al., 1992, A\&A 259, L19

Quirrenbach A. Mozurkewich D., Armstrong T., et al., 1993, ApJ 406, 215

Quirrenbach A., Hummel C.A., Buscher D.F., et al., 1993, ApJ 416, L25

Quirrenbach A., Mozurkewich D., Buscher D.F., et al., 1996, A\&A 312, 160

Quirrenbach A., 2001, Ann. Rev. Astron. Astrophys. 39, 353

Schwarzschild M., 1975, ApJ 195, 137

Segransan D., Kervella P., Forveille T., Queloz D., 2003, A\&A, in press

Shao M., Colavita M.M., 1992, Ann. Rev. Astron. Astrophys. 30, 457

Stee P., de Araujo F.X., Vakili F., et al., 1995, A\&A 300, 219

Stee Ph., Bittar J., 2001, A\&A 367, 532

Thompson R.R., Creech-Eakman M.J., van Belle G.T., 2002, ApJ 577, 447

van Belle G.T., Ciardi D.R., Thompson R.R., et al., 2001, ApJ 559, 1155

van Belle G.T., Thompson R.R., Creech-Eakman M.J., 2002, AJ 124, 1706

van Belle G.T., Ciardi D.R., Thompson R.R., Akeson R.L., this volume (a)

van Belle G.T., Thompson R.R., Creech-Eakman M.J., this volume (b)

Wittkowski M, Hummel C.A., Johnston K.J., et al., 2001, A\&A 377, 981

Wittkowski M., Schöller M., Hubrig S., et al., 2002, AN 323, 241

Wittkowski M., this volume

Wittkowski M., Richichi A., Correia S., Schöller M., this volume

Woitke P., this volume

Young J.S., Baldwin J.E., Boysen R.C., et al., 2000, MNRAS 315, 625

Young J.S., Baldwin J.E., Boysen, R.C., et al., 2000, MNRAS 318, 381 